\title{
Resonant thermal transport in semiconductor barrier structures
}

\author{
P. Hyldgaard \\ Department of Applied Physics, Chalmers University of Technology, SE-41296 Göteborg, Sweden.
}

(Dated: January 18, 2004)

\begin{abstract}
I report that thermal single-barrier (TSB) and thermal double-barrier (TDB) structures (formed, for example, by inserting one or two regions of a few Ge monolayers in $\mathrm{Si}$ ) provide both a suppression of the phonon transport as well as a resonant-thermal-transport effect. I show that high-frequency phonons can experience a traditional double-barrier resonant tunneling in the TDB structures while the formation of Fabry-Perot resonances (at lower frequencies) causes quantum oscillations in the temperature variation of both the TSB and TDB thermal conductances $\sigma_{\mathrm{TSB}}$ and $\sigma_{\mathrm{TDB}}$.
\end{abstract}

PACS numbers: $66.70 .+\mathrm{f}, 42.25 . \mathrm{Hz}, 44.10 .+\mathrm{i}, 63.20 .-\mathrm{e}$

The understanding of phonon transport in nanoscale heterostructured materials [1] is in an exciting development motivated in part by the search to improve both thermo-electric 2] and thermo-ionic [3] cooling. The interest also derives from the observation that nanostructure phonons exhibit nanoscale-transport, confinement, and quantization effects similar to those observed for electrons and photons. A significant suppression is observed [4, 5] in the in-plane thermal conductivity of heterostructures and is explained by interface scattering as a phonon Knudsen-flow effect [6, 7]. Similarly, the perpendicular thermal conductivity $\kappa_{\mathrm{SL}}$ of semiconductor superlattices shows a dramatic reduction [8, 9] (compared to the average bulk conductivities) that cannot be accounted for alone by the expected decrease [10] in the effective superlattice-phonon lifetime $\tau_{\mathrm{SL}}$. Instead, the strong reduction in $\kappa_{\mathrm{SL}} / \tau_{\mathrm{SL}}$ results from a pronounced miniband formation where the difference in materials hardness forces an increasing confinement of modes with a finite in-plane momentum to either the Si or Ge layers in the superlattice [11]. Finally, the phonon quantum-point-contact effect in nanoscale dielectric wires 12 shows that the phonon wave nature also directly affects the low-temperature phonon transport.

Here I extend the search for quantized thermaltransport effects in semiconductor nanostructures to finite temperatures. I focus on the phonon conduction across $\mathrm{Si} /$ few-Ge-monolayers/Si thermal singlebarrier (TSB) and corresponding $\mathrm{Si} / \mathrm{Ge} / \mathrm{Si} / \mathrm{Ge} / \mathrm{Si}$ thermal double-barrier (TDB) heterostructures. I document (i) a strong suppression of the phonon-transport thermal conductances $\sigma_{\mathrm{TSB}}$ and $\sigma_{\mathrm{TDB}}$, (ii) a traditional type of double-barrier resonant tunneling for high-frequency phonons in the TDB structures, and (iii) that phonon Fabry-Perot resonances [13] (at lower frequencies) produce a resonant-thermal-transport effect at finite temperatures in both the TSB and TDB structures (for sufficiently small Ge-barrier thicknesses). My focus is on the technology relevance rather than the low-temperature transport. Instead of the long-wavelength phonon analysis of Ref. [12], I therefore present a phonon-model calculation of the (resonant) thermal transport which remains
Thermal single-barrier (TSB) structure

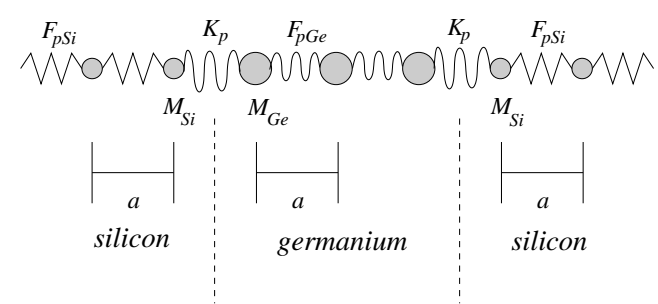

Thermal double-barrier (TDB) structure

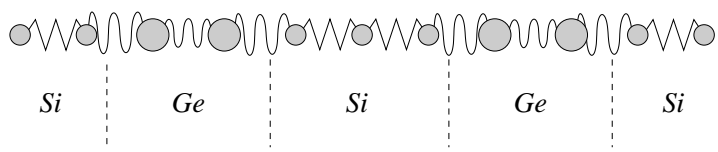

FIG. 1: Sample realization of thermal barrier structures in which even a few monolayers of a softer material (here germanium) serve to inhibit the otherwise effective (phonon) thermal transport in a surrounding hard materials (here $\mathrm{Si}$ ). The figure shows examples of both thermal single-barrier (TSB) and thermal double-barrier (TDB) heterostructures that exhibit a resonant-thermal transport effect. The figure also shows schematics of the phonon model used to calculate the finite-temperature variation of the TSB and TDB thermal conductances.

applicable at finite frequencies, includes the phase-space limitations imposed by total-internal reflection, and describes the important effect of increasing misalignment of the Si-/Ge-phonon dynamics as the in-plane momentum $q$ increases [11]. The prediction (iii) bridges the concept of thermal-transport quantization effects from the previous focus on low-temperature phonon transmission [12] to the finite-temperature nanostructure heat conduction for which I show that oscillations persist up to temperatures $\Theta \approx 50 \mathrm{~K}$.

To emphasize the potential technological relevance I first report a simple estimate for the thermalconductance suppression in two examples of TSB and TDB structures, Fig. 1, formed as a Si/triple-Gemonolayer/Si and as a $\mathrm{Si} / 2 \mathrm{Ge} / 3 \mathrm{Si} / 2 \mathrm{Ge} / \mathrm{Si}$ semiconductor heterostructure, respectively. I predict below a strong 
high-temperature suppression

$$
\sigma_{\mathrm{TSB}, \mathrm{TDB}} \sim \sigma_{K} \lesssim 10^{4} \mathrm{~W} / \mathrm{Kcm}^{2}
$$

specified by the single-Si/Ge interface Kapitza conductance [14] $\sigma_{\mathrm{K}}(\Theta) \leq \sigma_{\mathrm{K}}(\Theta \rightarrow \infty) \approx 10^{4} \mathrm{~W} / \mathrm{Kcm}^{2}$ (as also obtained in my phonon-model calculation). The thermalconductance estimate (1) results by describing the full dynamics of phonons that approach an individual singlebarrier/double-barrier structure (rather than moving in a superlattice) and therefore provides a more consistent description than the co-called effective conductance [9] $G_{\mathrm{SL}} \equiv \kappa_{\mathrm{SL}}(d) /(d / 2) \sim 10^{5} \mathrm{~W} / \mathrm{Kcm}^{2}$ extracted [9] from measurements at $(\Theta=200 \mathrm{~K})$ of the thermal conductivity $\kappa_{\mathrm{SL}}(d)$ in $\mathrm{Si} / \mathrm{Ge}$ superlattices with a nanoscale periodicity $d$. The estimate (11) shows that repeating the TSB or TDB formation every $50 \mathrm{~nm}$ provides a reduction of the effective thermal conductivity $\kappa$ to the value of a $\mathrm{Si} / \mathrm{Ge}$ alloy $\kappa_{\text {alloy }} \approx 5 \mathrm{~W} / \mathrm{Km}$ (repetition every $5 \mathrm{~nm}$ would be needed if the limit $\sigma_{\mathrm{TSB}} \lesssim G_{\mathrm{SL}}$ applies).

The TSB and TDB structures also give rise to a resonant-thermal-transport effect which is observable at finite temperatures $(\Theta \lesssim 50 \mathrm{~K})$. Fig. 1 shows a schematics of the phonon model which I here solve to calculate the phonon tunneling and the resulting thermal conductance across both a single interface and across the repeated interfaces in the TSB and TDB structures. I assume a shared silicon (germanium) lattice constant $a$, the atomic masses $M_{\mathrm{Si}(\mathrm{Ge})}$, and intra-silicon (intra-germanium) force constants $F_{p ; \mathrm{Si}(\mathrm{Ge})}$ and inter-layer coupling constant $K_{p} \equiv\left(F_{p: \mathrm{Si}} F_{p ; \mathrm{Ge}}\right)^{1 / 2}$ specified by the materials sound velocities [1]. The tunneling [15] of phonon modes that are polarized and propagating in the perpendicular $\hat{z}$ direction is well described within such a one-dimensional lattice model. I refer to our previous investigation of superlattice thermal transport [1] for a description of how I include the effects of a finite in-plane momentum within a simple-cubic model by adding in-plane force constants $F_{t ; \mathrm{Si}(\mathrm{Ge})}$ and corresponding characteristic frequencies $\Omega_{p, t ; \mathrm{Si}(\mathrm{Ge})} \equiv\left(4 F_{p, t ; \mathrm{Si}(\mathrm{Ge})} / M_{\mathrm{Si}(\mathrm{Ge})}\right)^{1 / 2}$; Fig. 1 of Ref. 11]. The set of force constants $F_{p, t ; \mathrm{Si}(\mathrm{Ge})}$ also specifies the phonon-transport contributions from $\xi_{\hat{x}, \hat{y}} \| \hat{x}, \hat{y}$ polarized heterostructure phonons [16] and these modes are, of course, also included in the transport calculation. Below I limit the formal discussion to the contributions from $\xi_{p} \| \hat{z}$-polarized modes which for a given in-plane momentum $\vec{q}$ can be characterized by the dimensionless in-plane energy measure [11] $0<\alpha_{\vec{q}} \equiv$ $\left[2-\cos \left(q_{x} a\right)-\cos \left(q_{y} a\right)\right]<4$. This measure is, like the frequency, conserved [1] across the heterostructure interfaces in this phonon-transport model study.

Figure 2 reports my calculations of the phonon transmission in the single-interface, TSB, and TDB structures. Starting from the model equations of motion for every atom and adapting the approach of Ref. 14], I determine the single-interface, TSB, and TDB transmission probabilities $T_{\mathrm{K}, \mathrm{TSB}, \mathrm{TDB}}\left(\omega, \alpha_{\vec{q}}\right)$ as a function of the

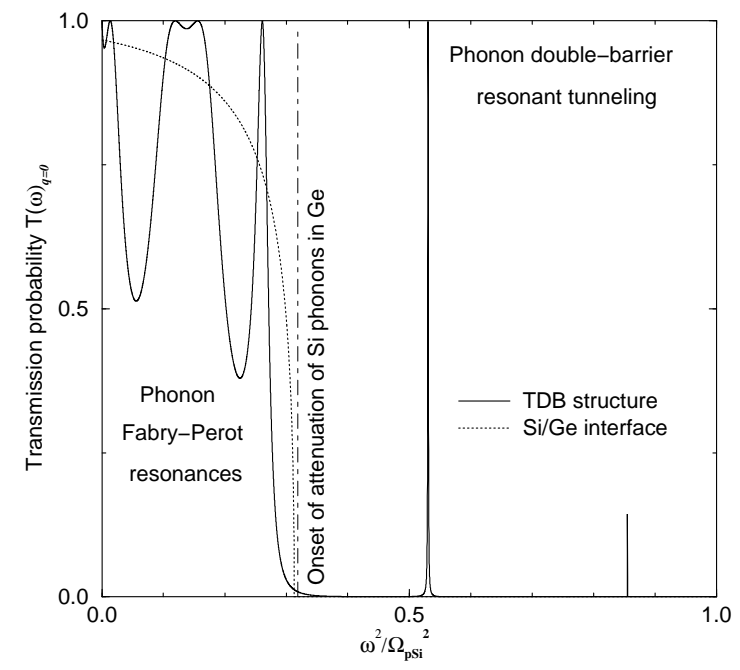

FIG. 2: Comparison of calculated phonon transmission probability (evaluated at in-plane momentum $q=0$ ) for the $\mathrm{Si} / \mathrm{Ge}$ thermal double-barrier structure, Fig. 1 (solid curve) and for the individual Si/Ge interface (dotted curve). The vertical line identifies the frequency-squared value above which incoming Si phonons become attenuated in Ge. The TDB structure is seen to support a traditional type of double-barrier resonant tunneling above this limit (when the Ge-layers represent actual barriers) but also multiple Fabry-Perot resonances (at lower frequencies) when incoming phonons experience a partial transmission at each of the individual Si/Ge interfaces. Note that the TDBs (and TSBs) naturally become completely transparent as $\omega \rightarrow 0$.

phonon frequency and (conserved) in-plane momentum $q$. The figure reports a comparison of $T_{\mathrm{K}}\left(\omega, \alpha_{\vec{q}=0}\right)$ and $T_{\mathrm{TDB}}\left(\omega, \alpha_{\vec{q}=0}\right)$ and identifies the onset (vertical dasheddotted line) when incoming Si phonons become attenuated in the Ge barriers. The figure documents that the TDB structure supports a traditional type of phonon (double-barrier) resonant tunneling at high frequencies $\left(\omega_{>}\right)$when the phonon dynamics in Ge is attenuated [and $T_{\mathrm{K}}\left(\omega_{>}\right)=0$ ]. The figure also illustrates that both the TDB and TSB structures support a number of FabryPerot resonances at lower frequencies $\left(\omega_{<}\right)$when the individual interfaces provide partial transmission [i.e., when $\left.0<T_{\mathrm{K}}\left(\omega_{<}\right)<1\right]$.

To clarify the nature and measurability of the high-frequency resonant tunneling, I summarize the model calculations of the phonon dynamics at general in-plane momentum, $\alpha_{\vec{q}} \neq 0$. I note that bulk silicon (germanium) phonon propagation at a given $\alpha_{\vec{q}}$ requires that the frequency square exceeds $\omega_{\mathrm{Si}(\mathrm{Ge}), \min }^{2}\left(\alpha_{\vec{q}}\right) \equiv \Omega_{t ; \mathrm{Si}(\mathrm{Ge})}^{2}\left(\alpha_{\vec{q}} / 2\right)$ but remains bounded by $\omega_{\mathrm{Si}(\mathrm{Ge}), \max }^{2}\left(\alpha_{\vec{q}}\right) \equiv \Omega_{t ; \mathrm{Si}(\mathrm{Ge})}^{2}\left(\alpha_{\vec{q}} / 2\right)+\Omega_{p ; \mathrm{Si}(\mathrm{Ge})}^{2}[11]$. The finite silicon/germanium acoustic mismatch ensures that $\omega_{\mathrm{Si}, \min [\max ]}\left(\alpha_{\vec{q}}\right)>\omega_{\mathrm{Ge}, \min [\max ]}\left(\alpha_{\vec{q}}\right)$. Phonon propagation (i.e., absence of attenuation) in both silicon and germa- 
nium layers thus effectively requires that

$$
\omega_{\mathrm{Si}, \min }^{2}\left(\alpha_{\vec{q}}\right)<\omega^{2}<\omega_{\mathrm{Ge}, \max }^{2}\left(\alpha_{\vec{q}}\right),
$$

since this is the condition for an incoming silicon phonon to avoid total internal reflection at an individual $\mathrm{Si} / \mathrm{Ge}$ interface (as formulated in the present model study). The condition (2) is a severe phase-space restriction which, for example, becomes impossible to satisfy at $\alpha_{\vec{q}}>2$ in $\mathrm{Si} / \mathrm{Ge}$ structures [1]. For incoming Si phonons with a frequency $\omega$ above the onset of Ge attenuation, the model study yields a strong exponential decay $1 / \gamma_{\mathrm{Ge}} \sim a$, a vanishing single-barrier transmission $\left(T_{\mathrm{TSB}} \rightarrow 0\right)$, and the strongly peaked double-barrier resonant-tunneling transmission $T_{\mathrm{TSB}}$ that is illustrated in Fig. 2. The highfrequency resonant tunneling may become observable 15. if it is possible to design a frequency-selective source of $\mathrm{THz}$ phonons [17]. However, for a thermal distribution $N(\omega, \Theta)$ of incoming $\mathrm{Si}$ phonons at a relevant elevated temperature $\Theta$, I find the phase-space contribution from the (high-frequency) resonant tunneling, Fig. 2, too small to directly affect the TDB thermal conductance.

In contrast, I find that the phonon Fabry-Perot resonances do produce observable finite-temperature quantization effects in the TDB and TSB heat conduction, Fig. 3. From the calculated phonon transmission probabilities I determine (adapting Ref. 14]) the single-Si/Ge interface and thermal-barrier conductances

$$
\begin{aligned}
\sigma_{\mathrm{K}, \mathrm{TSB}, \mathrm{TDB}} & =\sum_{m} \int \frac{d^{2} q}{(2 \pi)^{2}} \times \\
{\left[\int_{\omega_{\min }}^{\omega_{\max }} \frac{d \omega}{2 \pi}\right.} & \left.\hbar \omega T_{\mathrm{K}, \mathrm{TSB}, \mathrm{TDB}}\left(\omega ; \alpha_{\vec{q}}\right)\left(\frac{d N}{d \Theta}\right)\right]_{m}
\end{aligned}
$$

by summing up the contributions at different modes $m=\xi_{p}, \xi_{\hat{x}, \hat{y}}$ and in-plane momenta $q$. In the result (3) $\omega_{\min }$ and $\omega_{\max }$ represent a shorthand for the corresponding frequency-integration limits $\omega_{\mathrm{Si}, \min }\left(\alpha_{\vec{q}}\right)$ and $\omega_{\mathrm{Si}(\mathrm{Ge}), \max }\left(\alpha_{\vec{q}}\right)$. I stress that the condition (2) must be absolutely satisfied to obtain a transport contribution to the interface conductance $\sigma_{\mathrm{K}}$ and effectively satisfied for the TSB and TDB conductances $\sigma_{\mathrm{TSB}, \mathrm{TDB}}$ (however, all TSB and TDB-transport contributions are retained in the calculations reported here).

The top panel of Fig. 3 illustrates the general validity of the "classical" thermal-conductance approximation, Eq. (1), and documents the additional temperature variation produced by the low-energy Fabry-Perot resonances. The panel shows that the thermal-barrier conductance $\sigma_{\mathrm{TSB}}$ (solid curve) at all temperatures is comparable to and generally smaller than the individual Si/Ge-interface conductance $\sigma_{\mathrm{K}}$ (dotted curve).

The insert panel compares the corresponding phonon transmission probability $T_{\mathrm{TSB}}(\omega)$ (calculated for $\mathrm{Si} / 3 \mathrm{Ge} / \mathrm{Si}$ at $\vec{q}=0)$ and the single-Si/Ge-interface transmission (dotted curve). The insert motivates the

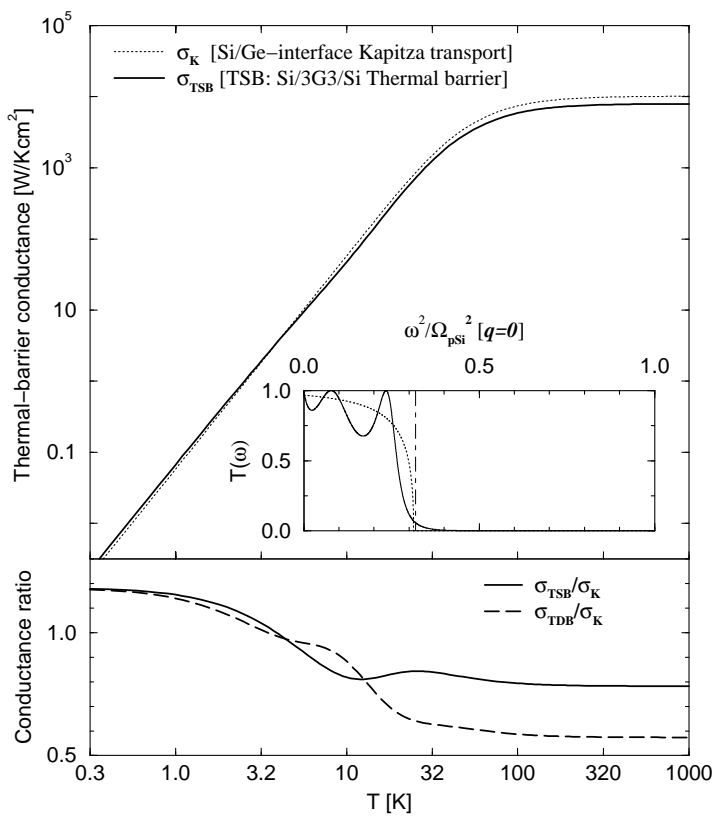

FIG. 3: Temperature dependence of (perpendicular) thermal conductance $\sigma_{\mathrm{TSB}}$ (solid curve) for a $\mathrm{Si} / 3 \mathrm{Ge} / \mathrm{Si} \mathrm{TSB}$ heterostructure compared with the $\mathrm{Si} / \mathrm{Ge}$-interface Kapitza conductance $\sigma_{K} \equiv \sigma_{\mathrm{Si} / \mathrm{Ge}}$ (dotted curve). The thermal barrier transport is effectively subject to similar severe phasespace restrictions (enforced by total internal reflection) as the single-interface conductance and can thus be estimated $\sigma_{\mathrm{TSB}}(T) \sim \sigma_{\mathrm{K}}(T)$. The insert panel compares the TSB phonon transmission probability $T_{\mathrm{TSB}}(\omega) \equiv T_{\mathrm{TSB}}\left(\omega ; \alpha_{q}=0\right)$ (solid curve) and the single-Si/Ge transmission probability (dotted curve) at zero in-plane momentum, $q=0$. The barrier transmission is seen to be essentially eliminated above the frequency (vertical dashed-dotted line) when the incoming phonon must tunnel across the Ge barrier layers. At the same time, the barrier transmission $T_{\mathrm{TSB}}$ also shows clearly defined low-energy phonon Fabry-Perot resonances (similar to those of the $T_{\mathrm{TDB}}$, Fig. 2) produced within the $3 \mathrm{Ge}$ barrier. The bottom panel documents how the set of lower-energy Fabry-Perot resonances produce quantum oscillations in the TSB and TDB conductance ratios $\sigma_{\mathrm{TSB}} / \sigma_{\mathrm{K}}$ (solid curve) and $\sigma_{\mathrm{TDB}} / \sigma_{\mathrm{K}}$ (dashed curve).

estimate (11) by emphasizing that (a) the thermal singlebarrier transport is effectively restricted to incoming Si modes that satisfy the condition (2) (exactly as was found for the TDB transport, Fig. 2) and (b) the singleinterface transmission (dotted curve) approximates the average thermal-barrier transmission

$$
\left\langle T_{\mathrm{TSB}}(\omega)\right\rangle \sim\left\langle T_{\mathrm{K}}(\omega)\right\rangle \approx T_{\mathrm{Si} / \mathrm{Ge}}^{0} \equiv \frac{4 Z_{\mathrm{Si}} Z_{\mathrm{Ge}}}{\left(Z_{\mathrm{Si}}+Z_{\mathrm{Ge}}\right)^{2}},
$$

specified by the differences in acoustic impedances, $Z_{\mathrm{Ge}} / Z_{\mathrm{Si}} \approx 1.5[15]: T_{\mathrm{Si} / \mathrm{Ge}}^{0} \approx 0.95$. The observations (a) and (b) complete the argument (proof) for the "classical" thermal-conductance estimate (11).

The bottom panel of Fig. 3 documents that the phonon Fabry-Perot resonances refine the classical estimate (11) 


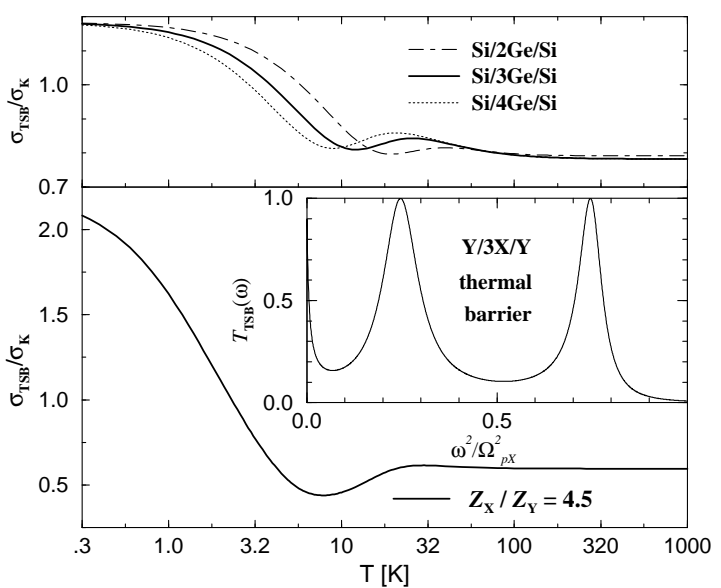

FIG. 4: Robustness (top panel) and enhancement (bottom panel) of the resonant-thermal-transport effects. The figure reports calculations of the thermal conductance ratio $\sigma_{\mathrm{TSB}} / \sigma_{\mathrm{K}}$ for both a set of $\mathrm{Si} / \mathrm{Ge} / \mathrm{Si}$ systems with different numbers of layers in the Ge barrier, and a fictitious $\mathrm{X} / 3-\mathrm{Y}$-monolayer/X TSB structure with the significantly larger acoustic-impedance ratio $Z_{X} / Z_{Y} \approx 4.5$ [18]. The top panel documents that the predicted TSB resonantthermal-transport effect is robust as the set of different barrier thicknesses produce qualitatively identical oscillations in the temperature variation of the TSB thermal-conductance ratio. The insert and bottom panels show that increasing the ratio of acoustic impedances causes much stronger Fabry-Perot resonances in the TSB transmission and an amplification of the thermal-conductance oscillations with temperature.

in producing resonant-thermal-transport effects. These arise at higher temperatures and in a more general and technologically relevant group of structures than the previously investigated case of dielectric quantum wires, Ref. [12]. Specifically, the bottom panel of Fig. 3 details quantum oscillations in the temperature variations for both $\sigma_{\mathrm{TSB}} / \sigma_{\mathrm{K}}$ (solid curve) and $\sigma_{\mathrm{TDB}} / \sigma_{\mathrm{K}}$ (dashed curve). In both structures the thermal-conductance oscillations enhance as $T \rightarrow 0$ where the $T_{\mathrm{TSB}}(\omega)$ and $T_{\mathrm{TDB}}(\omega)$ variation increases in relative importance.

Finally, Fig. 4 emphasizes that the resonant-thermaltransport effects are robust against variations in the barrier thickness and enhance with an increasing ratio of the acoustic impedances of the TSB and TDB heterostructures. The figure reports calculations of the thermalconductance variation both for a set of $\mathrm{Si} / \mathrm{Ge} / \mathrm{Si}$ systems and in a fictitious TSB structure 18] X/3Y/X where $Z_{X} / Z_{Y} \approx 4.5$. The latter change produces a barrier transmission (insert panel) where the FabryPerot resonances cause a much more dramatic deviation from the average transmission approximated by the longwavelength single-interface estimate (4), $\left\langle T_{\mathrm{K}}(\omega)\right\rangle \sim 0.6$.

In summary, I have investigated the phonon transport perpendicular to the interfaces of (silicon/triplegermanium-layer/silicon) thermal single-barrier (TSB) and corresponding thermal double-barrier (TDB) structures. I document a strong suppression of the finite- temperature heterostructure thermal conductances $\sigma_{\mathrm{TSB}}$ and $\sigma_{\mathrm{TDB}}$ which approximately are limited by the conductance $\sigma_{\mathrm{K}}$ of an individual $\mathrm{Si} / \mathrm{Ge}$ interface. In addition, I predict quantum oscillations in the thermalconductance ratios $\sigma_{\mathrm{TSB}} / \sigma_{K}$ and $\sigma_{\mathrm{TDB}} / \sigma_{K}$ which arise from phonon Fabry-Perot resonances trapped in the central barrier or double-barrier region, respectively.

Discussions with G. D. Mahan are gratefully acknowledged. This work was supported by the Swedish Foundation for Strategic Research (SSF) through ATOMICS.

[1] D. G. Cahill et al, J. Appl. Phys. 93, 793 (2003).

[2] L. D. Hicks and M. S. Dresselhaus, Phys. Rev. B 47, 12727 (1993); G. D. Mahan and H. B. Lyon, Jr., J. Appl. Phys. 76, 1899 (1994); J. O. Sofo and G. D. Mahan, Appl. Phys. Lett. 65, 2690 (1994); D. A. Broido and T. L. Reinecke, Phys. Rev. B 51, 13797 (1995).

[3] G. D. Mahan and L. M. Woods, Phys. Rev. Lett. 80, 4016 (1998).

[4] T. Yao, Appl. Phys. Lett. 51, 1798 (1987).

[5] X. Y. Yu, G. Chen, A. Verma, and J. S. Smith, Appl. Phys. Lett. 67, 3554 (1995).

[6] P. Hyldgaard and G. D. Mahan, in Thermal conductivity (Technomic Publishing Company, Inc., Lancaster, Pennsylvania, 1996), Vol. 23, pp. 172-182.

[7] G. Chen, J. Heat Trans. 119, 220 (1997).

[8] G. Chen et al, Jour. of Heat Transf. 116, 325 (1994); W. S. Capinski and H. J. Maris, Physica B 219, 220, 699 (1996).

[9] S. M. Lee, D. G. Cahill, R. Venkatasubramanian, Appl. Phys. Lett. 70, 2957 (1997).

[10] S. Y. Ren and J. D. Dow, Phys. Rev. B 25, 3750 (1982); A. Balandin and K. L. Wang, Phys. Rev. B 58, 1544 (1998).

[11] P. Hyldgaard and G. D. Mahan, Phys. Rev. B 56, 10754 (1997); The value of $\kappa_{S L} / \tau_{S L}$ decreases as the superlattice periodicity $d$ increases (unpublished).

[12] L.G.C. Rego and G. Kirczenow, Phys. Rev. Lett. 81, 232 (1998); K. Schwab et al, Nature 404, 974 (2000).

[13] Phonon Fabry-Perot resonances arise from partial reflection at the interfaces in the barrier structures. The corresponding photon concept is discussed, for example, in M. Young, "Optics and lasers" (Springer-Verlag, Berlin, 1992) p. 136.

[14] S. Pettersson and G. D. Mahan, Phys. Rev. B 42, 7386 (1990); R. J. Stoner and H. J. Maris, ibid. 48, 16373 (1993).

[15] V. Narayanamurti et al, Phys. Rev. Lett. 43, 2012 (1979); S. Mizuno and S. I. Tamura, Phys. Rev. B 45734 (1992).

[16] I fit the values of $F_{p, t ; \mathrm{Si}}$ and $F_{p, t ; \mathrm{Ge}}$ to match the (longwavelength) [100] sound-velocities of the Si and Ge longitudinal and transverse modes as described in Ref. [11].

[17] P. Hyldgaard, Mater. Sci. Eng., C 23, 243 (2003); P. Hyldgaard, Low Temp. Phys. 27, 585 (2001); V. Narayanamurti, Science 213, 717 (1981).

[18] The impedance ratio $Z_{\mathrm{X}} / Z_{\mathrm{Y}} \approx 4.5$ is very large but would characterize the phonon transport in a hypothetical $\mathrm{Mg}$ /triple-Hf-monolayer/Mg structure. 